\title{
On Kempton's Generalization of the Negative Binomial Distribution
}

\author{
S.H. Ong', Y.C. Low ${ }^{2 *}$ \\ ${ }^{1}$ Department of Actuarial Science and Applied Statistics, UCSI University, 56000 Kuala Lumpur, Malaysia \\ ${ }^{2}$ Department of Computing and Information Systems, Sunway University, 47500 Selangor, Malaysia \\ *Corresponding Author: yehchingl@sunway.edu.my, ongsh@ucsiuniversity.edu.my \\ Received: 14 ${ }^{\text {th }}$ May $2019 \quad$ Revised: $4^{\text {th }}$ July $2019 \quad$ Accepted: $28^{\text {th }}$ Aug 2019 \\ DOI: https://doi.org/10.22452/mjs.vol38no2.5
}

\begin{abstract}
This paper examines the properties of and applications to empirical modelling as well as computation of probabilities of Kempton's generalization of the negative binomial and log-series distributions. Important properties of infinite divisibility and unimodality have been derived. To facilitate computation of the complicated probabilities, practical implementation of the three-term probability recurrence relations is presented. While the generalization of the negative binomial and log-series distributions have been formulated to fit extremely long-tailed count data, the versatility of this generalized negative binomial distribution to fit short-tailed and long-tailed data is illustrated here.
\end{abstract}

Keywords: infinite divisibility, mixed Poisson, numerical stability, three-term recurrence, unimodality

\section{INTRODUCTION}

The statistical modelling and analysis of species abundance is an area of great interest and importance in ecology since the seminal paper by Fisher, Corbet and Williams (1943); see for example, Dallas et al. (2017) and Weber et al. (2017). To model species abundance, Fisher, Corbet and Williams (1943) showed that the number of species $s_{i}$, with $i$ individuals in a sample, follows a log-series distribution. This was modelled as follows: Assume that for some species with a given abundance, the number of individuals follows a Poisson distribution, and the abundances among species follow a gamma distribution. The individuals are then seen to follow the negative binomial (NB) distribution. For modelling the species abundance, the NB distribution is truncated at zero since the number of species is unknown. Fisher, Corbet and Williams (1943) noticed that the shape parameter of gamma distribution (mixing distribution) should be allowed to go to 0 while the number of species approaches infinity with their product going to a positive constant $C$.

Sharing a similar idea, Kempton (1975) considered the generalization of the log-series distribution (GLSD) by deriving the generalization of $\mathrm{NB}$ distribution, which is also known as the full beta model (FBM) (see also Holla and Bhattacharya, 1965). Kempton (1975) obtained the FBM as a mixed Poisson distribution with mixing distribution having a probability density function (pdf) given as 


$$
g(\lambda)=\frac{b^{p} \lambda^{p-1}}{B(p, q)(1+b \lambda)^{p+q}} .
$$

Then the resultant distribution has the following probability mass function (pmf)

$$
\begin{aligned}
P(k) & =\frac{1}{B(p, q)} \int_{0}^{\infty} e^{-\lambda} \frac{\lambda^{k}}{k !} \frac{b^{p} \lambda^{p-1}}{(1+b \lambda)^{p+q}} d \lambda, \\
& =\frac{\Gamma(p+k)}{k ! B(p, q) b^{q}} \psi(p+q, q+1-k ; 1 / b), \quad k=0,1,2, \ldots
\end{aligned}
$$

The form (1.2) is derived from the transformation (Erdélyi et al, 1953, Vol. I, p. 255) of

$$
\psi(a, c ; x)=x^{1-c} \psi(1+a-c, 2-c ; x)
$$

Note that (1.1) is the beta prime distribution with scale parameter $1 / b$. It is also known as the inverted beta distribution and is a Pearson Type VI distribution.

The GLSD has pmf

$$
\begin{aligned}
& \qquad \begin{aligned}
P(k) & =\frac{\alpha}{k !} \int_{0}^{\infty} \frac{x^{k-1} e^{-x}}{(1+b x)^{q}} d x, \\
& =\frac{\alpha}{k b^{q}} \psi(q, 1-k+q ; 1 / b), \quad k \geq 1
\end{aligned} \\
& \text { where } \alpha^{-1}=\sum_{r=1}^{\infty} \int_{0}^{\infty} x^{r-1} e^{-x}(1+b x)^{-q} d x / r !
\end{aligned}
$$

Kempton's (1975) interest was in the derivation of the GLSD for modelling species abundance where the frequency count data is characterized by an extremely long tail. However, there was no discussion on the properties of the full beta model. This full beta model (FBM) as a mixed Poisson distribution is of interest on its own because mixed Poisson distributions is an important class of distributions for the statistical analysis of over-dispersed (variance exceeding the mean) count data. A comprehensive survey of mixed Poisson distributions was given by Karlis and Xekalaki (2005), and Gupta and Ong (2005) reviewed the applications of these mixed distributions to very long-tailed count data.
Ong (1995) and Ong and Muthaloo (1995) examined the computations of probabilities by threeterm recurrence relations of Kempton's (1975) GLSD and other related distributions (Tripathi, Gupta and White, 1987). These distributions have the interesting feature of being able to fit very long-tailed frequency data. The probability recurrence relations derived by Ong (1995) for the FBM and the GLSD to facilitate computations are respectively 


$$
\begin{aligned}
& k(k+1) P(k+1)=k\left(k-q-b^{-1}\right) P(k)+(k+p-1) P(k-1) / b, \\
& k(k+1) P(k+1)=k\left(k-q-b^{-1}\right) P(k)+(k-1) P(k-1) / b,
\end{aligned}
$$

for $k \geq 2$. The stability of the recurrence formulae in computation was discussed. A recurrence is said to be stable if the round-off errors do not grow relative to the size of the required function. Recurrence relations (1.3) and (1.4) are stable in the forward direction at a certain value of $k$ onwards (see Ong, 1995, p. 265). In other words, starting the recurrence from this $k$ onwards will give reliable computed probabilities compared to starting from $k=0$. This feature of the recurrence relations (1.3) and (1.4) makes their implementation in a computer routine difficult since this particular $k$ has to be determined before the recurrence formula can be used. This problem of determining $k$ such that (1.3) and (1.4) can be used safely becomes acute if it is employed in a numerical optimization of the (log) likelihood function. In this situation, a value of $k$ has to be determined for every point $(p, q, b)$ of the parameter space.

The objectives of this paper are to (i) derive important properties of the generalized NB distribution like infinite divisibility and unimodality, (ii) consider the implementation of the recurrence relations (1.3) and (1.4) based upon a heuristic approach of selecting $k$ and (iii) illustrate the versatility of the FBM in fitting not only very long-tailed data (Kempton, 1975, p. 36) but also short-tailed and moderately long-tailed data. Since (1.3) and (1.4) are similar, the discussion would be restricted to the recurrence relation (1.3).

The organization of the paper is as follows. Section 2 gives several probabilistic properties of the FBM. A heuristic method for testing forward stability of three-term recurrence relation for probabilities is presented in Section 3. Section 4 shows the versatility and superiority of the FBM in empirical modelling of count data. Concluding remarks are given in Section 5.

\section{Properties of the Full Beta Model}

In this section we give the properties of the FBM. These properties are consequences of the generalized NB as a mixed Poisson distribution. To facilitate the presentation, some properties of the beta prime distribution with pdf (1.1) are first presented.

\subsection{Some properties of beta prime distribution}

The beta prime distribution has $k$-th moment given by

$$
E\left[X^{k}\right]=\frac{B(p+k, q-k)}{B(p, q) b^{k}}, \quad q \geq k .
$$

The moment generating function (mgf) is

$$
M_{X}(t)=E\left[t^{X}\right]=e^{-t^{1 / b}} \frac{\Gamma(p+q)}{\Gamma(q)} G_{1,2}^{2,0}\left(\begin{array}{c}
p+q \\
q, 0
\end{array} \mid-t^{1 / b}\right)
$$


where $G_{\alpha, \beta}^{m, n}$ is the Meijer G-function defined by

$$
G_{p, q}^{m, n}\left(\begin{array}{l}
a_{1}, \ldots, a_{p} \\
b_{1}, \ldots, b_{q}
\end{array} \mid x\right)=\frac{1}{2 \pi i} \int_{C} \frac{\prod_{j=1}^{m} \Gamma\left(b_{j}+t\right) \prod_{j=1}^{n} \Gamma\left(1-a_{j}-t\right)}{\prod_{j=n+1}^{m} \Gamma\left(a_{j}+t\right) \prod_{j=m+1}^{n} \Gamma\left(1-b_{j}-t\right)} x^{-t} d t
$$

The definitions of infinite divisibility and log-concavity are presented next.

Infinitely divisible distributions constitute an important family of distributions in modelling (Steutel, 1983). A distribution function $F$ is said to be infinitely divisible if, for every $n \in N$,

$$
X^{=} X_{n, 1}+X_{n, 2}+\ldots+X_{n, n}
$$

where $F$ is the distribution of $X$ and $X_{n, 1}, X_{n, 2}, \ldots, X_{n, n}$ are independent and identically distributed.

A function $f$ is said to be logconcave on the interval $I$ if the function $\log (f)$ is a concave function on $I$. Logconcave distributions and the various preservation properties of log-concavity have important applications in many areas (Bagnoli and Bergstrom, 2005; Walther, 2009).

The beta prime distribution has the following important properties, summarized in the following theorems.

Theorem 1: The beta prime distribution is infinitely divisible and log-concave.

Proof: For infinitely divisibility see Steutel and van Harn (2003, Appendix B, section 3).

Log-concavity is proven in Borzadaran and Borzadaran (2011, Table 2, p. 209) for the Pearson Type VI distribution with restrictions on the parameters.

Various reliability properties of the beta prime distribution are a consequence of the log-concavity property (see Borzadaran and Borzadaran, 2011, p. 205). In particular, the beta prime distribution is strongly unimodal.

\subsection{Properties of the full beta model}

Since the FBM is a mixed Poisson distribution, a number of properties can be inferred directly from the mixing distribution, that is, the beta prime distribution. The probability generating function (pgf) of the FBM is obtained from the mgf of the mixing distribution by the formula about mixed Poisson distribution (see, for example, Karlis and Xekalaki, 2005, p.38) and is given as

$$
G_{X}(z)=M_{\Lambda}(z-1) .
$$


We get, from (2.2),

$$
G_{X}(z)=e^{-(z-1)^{1 / b}} \frac{\Gamma(p+q)}{\Gamma(q)} G_{1,2}^{2,0}\left(\begin{array}{c}
p+q \\
q, 0
\end{array} \mid-(z-1)^{1 / b}\right) .
$$

The $r$ th factorial moment $\mu_{(r)}$ of the FBM corresponds to the $r$ th moment of the beta prime distribution (2.1)

$$
\mu_{(r)}=\frac{B(p+r, q-r)}{B(p, q) b^{r}}, \quad q \geq r
$$

This formula has been derived by Ong and Muthaloo (1995, p. 936) through the evaluation of an integral.

Theorem 2: The FBM is infinitely divisible.

Proof: If the mixing distribution in a mixed Poisson distribution is infinitely divisible, the mixed Poisson distribution is also infinitely divisible (Maceda, 1948).

Furthermore, Theorem 2 connects a mixed Poisson distribution to a compound Poisson distribution.

Theorem 3: The FBM is a compound Poisson distribution with GLSD whose pgf is

$$
Q(z)=1-\frac{1}{\theta}\left\{-\log G_{X}(z)\right\}, \quad \theta=-\log G_{X}(0)
$$

Proof: A discrete infinitely divisible distribution is a Poisson-stopped sum (compound Poisson) distribution (Feller,
1968). A compound Poisson distribution has $\mathrm{pgf}$ of the form (Steutel and van Harn, 2003, Theorem 3.2)

$$
G_{X}(z)=\exp (\theta(Q(z)-1))
$$

where $Q(z)$ is the pgf of the summand distribution (cluster distribution). For the FBM, based on (2.3) and equation (3.4) of Steutel and van Harn (2003), $Q(z)$ is given by

$$
Q(z)=1-\frac{1}{\theta}\left\{-\log G_{X}(z)\right\}, \quad \theta=-\log G_{X}(0) .
$$

This is the pgf of Kempton's generalized log-series distribution, GLSD.

Theorem 4: The FBM is unimodal.

Proof: If the mixing distribution in a mixed Poisson distribution is unimodal, the mixed Poisson distribution is also unimodal (Holgate, 1970).

\section{Computation of Probabilities by Recurrence Relation}

There are a number of heuristic approaches (Press et al., 2007) to determine the stability of the recurrence relations in the forward direction. One easy-to-implement method is the following: Consider the recurrence 
relation which may be started (first) with initial values 1 and 0 and then (second) with 0 and 1 (Press et al., 2007, p. 220). Ten to twenty terms are generated for each of the two starting conditions. If the differences between the corresponding members of the two sequences are of absolute value less than 10, then the recurrence is stable. If the differences increase slowly, the recurrence is mildly unstable but tolerable. Note that in this method of testing stability, the actual starting values of the functions are not required. This approach may be used to determine $k$ such that (1.3) is stable from this $k$ onwards at a particular point $(p, q, b)$ in the parameter space. To illustrate this heuristic approach, a numerical example is given in Table 1 below. The value of $k$ for each column is the starting value of the recurrence formula and the entries in Table 1 are the absolute difference of the two generated sequences. Table 2 reproduced from Ong (1995, Table I) gives the actual values of the probabilities and values computed from different starting points of the recurrence relation (1.3). It is apparent from a comparison of Tables 1 and 2 that the heuristic rule is conservative for this example since at $k=4$ the recurrence relation (1.3) is already stable.

Table 1. Illustration of heuristic method for recurrence formula (1.3) with

\begin{tabular}{|c|c|c|c|c|c|}
\hline \multirow[b]{2}{*}{$k$} & \multicolumn{5}{|c|}{$p=2.42, q=5.02 b=0.072$} \\
\hline & 1 & 2 & 4 & 8 & 9 \\
\hline 0 & 1 & & . & & \\
\hline 1 & 25.76 & 1 & . & & \\
\hline 2 & 153.11 & 13.55 & . & & \\
\hline 3 & 740.72 & 59.02 & . & & \\
\hline 4 & 2784.96 & 226.99 & 1 & & \\
\hline 5 & 8657.54 & 701.62 & 6.75 & & \\
\hline 6 & 22799.10 & 1850.85 & 18.61 & & \\
\hline 7 & 52018.42 & 4220.39 & 50.87 & & \\
\hline 8 & 104480.32 & 8478.76 & 114.59 & 1 & \\
\hline 9 & 187175.29 & 15188.00 & 231.33 & 3.03 & 1 \\
\hline 10 & 302245.26 & 24526.44 & 413.49 & 4.61 & 2.60 \\
\hline 11 & 443806.09 & 36012.70 & 668.44 & 8.10 & 3.55 \\
\hline 12 & 596984.88 & 48443.26 & 980.90 & 11.36 & 5.73 \\
\hline 13 & 740341.88 & 60075.49 & 1319.96 & 15.72 & 7.28 \\
\hline 14 & 851113.55 & 69064.70 & 1636.52 & 19.14 & 9.38 \\
\hline 15 & 911427.93 & 73958.51 & 1881.74 & 22.29 & 10.50 \\
\hline 16 & . & 74088.28 & 2014.78 & 23.63 & 11.48 \\
\hline 17 & . & . & 2018.54 & 23.87 & 11.31 \\
\hline 18 & . & $\ldots$ & 1898.54 & 22.29 & 10.80 \\
\hline$\cdot$ & & & $\cdot$ & $\cdot$ & $\cdot$ \\
\hline 21 & & & . & 13.23 & 6.26 \\
\hline
\end{tabular}


The heuristic method discussed above is easy to implement on a computer. This method has been applied over the parameter space $(p, q, b)$ of practical interest for $0<p, q, b<50$ in steps of 0.1 . It is found in general that (1.3) is stable at a starting point of $k \leq 10$ when $p, q, b<25$. If any of the parameters $p, q$ or $b$ is greater than 25 , the starting point is $k>10$. On the basis of this empirical evidence, the following approach may be adopted in computing probabilities of the FBM.
Test the recurrence relation (1.3) for stability at starting points $k=0,1,2, \ldots, t=10$. If the starting point is $k \leq 10$, compute $P(k)$ for $k<10$ by numerical integration or Monte Carlo integration (Ong et al., submitted) and use (1.3) for $k>10$; otherwise compute all the probabilities by numerical integration or by other methods.

Remark: In fitting short-tailed frequency data, choose $t$ smaller than 1 .

Table 2. Forward stability: Recurrence relation (1.3) (Ong, 1995)

\begin{tabular}{|c|c|c|c|c|}
\hline$k$ & $P^{(1)}(k)$ & $p^{(2)}(k)$ & $p^{(3)}(k)$ & $p^{(4)}(k)$ \\
\hline 0 & $0.43594736(-1)^{* *}$ & $0.43594736(-1)^{*}$ & & \\
\hline 1 & $0.72244871(-1)$ & $0.72244871(-1)^{*}$ & $0.72244871(-1)^{*}$ & \\
\hline 2 & $0.85726426(-1)$ & $0.85721075(-1)$ & $0.85726426(-1)^{*}$ & \\
\hline 3 & $0.88758952(-1)$ & $0.88789187(-1)$ & $0.88759027(-1)$ & \\
\hline 4 & $0.85540095(-1)$ & $0.85392469(-1)$ & $0.85539795(-1)$ & $0.85540095(-1)^{*}$ \\
\hline 5 & $0.79017278(-1)$ & $0.79571268(-1)$ & $0.79018457(-1)$ & $0.79017278(-1)^{*}$ \\
\hline 6 & $0.71070417(-1)$ & $0.69347405(-1)$ & $0.71066791(-1)$ & $0.71070417(-1)$ \\
\hline 7 & $0.62822133(-1)$ & $0.67358895(-1)$ & $0.62831693(-1)$ & $0.62822113(-1)$ \\
\hline 8 & $0.54898205(-1)$ & $0.45465451(-1)$ & $0.54876371(-1)$ & $0.54898205(-1)$ \\
\hline 9 & $0.47613877(-1)$ & $0.68405032(-1)$ & $0.47657750(-1)$ & $0.47613877(-1)$ \\
\hline 10 & $0.41097606(-1)$ & $0.38501577(-2)$ & $0.41019025(-1)$ & $0.41097607(-1)$ \\
\hline $\begin{array}{c}11 \\
\vdots\end{array}$ & $0.35370453(-1)$ & $0.95516290(-1)$ & $0.35497357(-1)$ & $0.35370453(-1)$ \\
\hline 37 & $0.11313668 \quad(-2)$ & $0.11392617(-2)$ & $0.11313836(-2)$ & $0.11313668 \quad(-2)$ \\
\hline 38 & $0.101706997(-2)$ & $0.10137907(-2)$ & $0.10170632(-2)$ & $0.101706997(-2)$ \\
\hline * & $\begin{array}{c}p=2.42, \quad q \\
\text { itial values for recurr }\end{array}$ & $\begin{array}{cr}.02, & b= \\
c e, \quad * * & 0.4359473\end{array}$ & 072 & $10^{-1}$ \\
\hline
\end{tabular}

$p^{(1)}(k)=$ probabilities computed by numerical integration

$p^{(2)}(k)=$ probabilities computed by (1.4) stating at $k=0$

$p^{(3)}(k)=$ probabilities computed by (1.4) starting at $k=1$

$p^{(4)}(k)=$ probabilities computed by (1.4) starting at $k=4$ 


\section{Examples of Data Fitting}

Kempton (1975, p.36) fitted the FBM to a very long-tailed frequency data set, that is, the species frequency distribution of insect catches. To exemplify the fit of the FBM to very short-tailed and moderately long-tailed frequency data, the following data sets have been considered:

(i) counts of red mites on apple leaves (Garman, 1951),

(ii) claims in automobile insurance (Bhati et al., 2015),

(iii) hospital stays (Gomez-Deniz and Calderın-Ojeda, 2015), and

(iv) number of sickness absences for men (Taylor, 1967).

The automobile insurance claims data is short-tailed whereas the data on number of sickness absences for men is a moderately long-tailed frequency data.
The parameters are estimated by maximum likelihood estimation via numerical maximization of the likelihood function. The results of the fit as judged by the chi-square values are presented in Tables 3 to 6 . For the counts of red mites, the FBM fit as well as the non-central negative binomial (NNB) distribution (Ong and Lee, 1979), which is another extension of the negative binomial distribution. Table 4 displayed the fits for the number of claims in automobile insurance and compared the FBM with the generalized Poisson-Lindley (GPL) distribution of Bhati et al. (2015). The FBM fit is clearly better. For the hospital stays data (Table 5) fitted by GomezDeniz and Calderın-Ojeda (2015) to a generalized geometric distribution, the FBM again fits much better. The FBM is compared to the Poisson-inverse Gaussian distribution (Sichel, 1971) for Taylor's data in Table 6; there is an overall improvement in fit.

Table 3. Counts of red mites on apple leaves $(\hat{p}=1.180, \hat{q}=38.729, \hat{b}=0.041)$

\begin{tabular}{ccccc}
\hline $\begin{array}{c}\text { No. of mites } \\
\text { per leaf }\end{array}$ & $\begin{array}{c}\text { Leaves } \\
\text { observed }\end{array}$ & $\begin{array}{c}\text { Negative } \\
\text { binomial }\end{array}$ & $\begin{array}{c}\text { NNB }(\lambda=2) \\
\text { (Ong and Lee, 1979) }\end{array}$ & FBM \\
\hline 0 & 70 & 64.49 & 69.98 & 69.94 \\
1 & 38 & 39.03 & 35.66 & 36.93 \\
2 & 17 & 20.96 & 20.75 & 19.68 \\
3 & 10 & 10.97 & 11.45 & 10.59 \\
4 & 9 & 5.66 & 6.06 & 5.75 \\
5 & 3 & 2.90 & 3.11 & 3.14 \\
6 & 2 & 1.48 & 1.55 & 1.74 \\
7 & 1 & 0.75 & 0.76 & 1.27 \\
$\geq 8$ & 0 & 0.76 & 0.68 & \\
\hline Total & 150 & 150.00 & 150.00 & 2.45 \\
\hline$\chi^{2}$ & & 2.93 & 2.45 & 0.9640 \\
\hline$p$-value & & 0.9387 & 0.9640 & \\
\hline
\end{tabular}


Table 4. Number of claims in automobile insurance $(\hat{p}=8.98, \hat{q}=3.38, \hat{b}=19.40)$

\begin{tabular}{ccccc}
\hline $\begin{array}{c}\text { Number of } \\
\text { claims }\end{array}$ & $\begin{array}{c}\text { Claims } \\
\text { Observed }\end{array}$ & NB & GPL (Bhati et al., 2015) & FBM \\
\hline 0 & 1563 & 1566.4 & 1564.50 & 1563.94 \\
1 & 271 & 261.5 & 264.25 & 267.54 \\
2 & 32 & 40.146 & 39.69 & 36.36 \\
3 & 7 & 5.99 & 5.59 & 5.57 \\
4 & 2 & 0.88 & 0.87 & 1.11 \\
\hline Total & 1875 & & & 1.64 \\
\hline$\chi^{2}$ & & 3.60 & 3.49 & 0.8016 \\
\hline$p$-value & & 0.4628 & 0.4794 &
\end{tabular}

Table 5. Hospital Stays Data $(\hat{p}=0.54, \hat{q}=3.91, \hat{b}=0.63)$

\begin{tabular}{ccccc}
\hline Number of days & $\begin{array}{c}\text { Days } \\
\text { Observed }\end{array}$ & NB & $\begin{array}{c}\text { Generalized Geometric } \\
\text { Gomez-Deniz and Calderín- } \\
\text { Ojeda, 2015) }\end{array}$ & FBM \\
\hline 0 & 3541 & 3544.63 & 3539.81 & 3539.73 \\
1 & 599 & 583.35 & 606.12 & 605.96 \\
2 & 176 & 177.5 & 161.2 & 165.08 \\
3 & 48 & 62.12 & 56.53 & 55.04 \\
4 & 20 & 23.35 & 23.06 & 21.18 \\
5 & 12 & 8.81 & 10.2 & 9.14 \\
6 & 5 & 3.52 & 4.72 & 4.34 \\
7 & 1 & 1.32 & 2.24 & 2.23 \\
8 & 4 & 0.44 & 1.08 & 1.22 \\
\hline Total & 4406 & \multicolumn{5}{c}{} \\
\hline$\chi^{2}$ & & 34.73 & 12.05 & 6.89 \\
\hline$p$-value & & 0.00003 & 0.1490 & 0.5485 \\
\hline
\end{tabular}

Table 6. Number of sickness absences $(\hat{p}=2.20, \hat{q}=6.14, \hat{b}=0.05)$

\begin{tabular}{cccc}
\hline $\begin{array}{c}\text { Number of } \\
\text { absences }\end{array}$ & $\begin{array}{c}\text { Observed } \\
\text { Frequency }\end{array}$ & $\begin{array}{c}\text { Poisson-inverse Gaussian } \\
\text { (Sichel, 1971) }\end{array}$ & FBM \\
\hline 0 & 31 & 20.1 & 28.03 \\
1 & 35 & 43.5 & 44.71 \\
2 & 55 & 57.3 & 52.32 \\
3 & 59 & 60.7 & 54.03 \\
4 & 49 & 57.7 & 52.22 \\
5 & 59 & 51.9 & 48.51 \\
6 & 41 & 45.3 & 43.94 \\
7 & 38 & 38.9 & 39.12 \\
8 & 32 & 33.3 & 34.43 \\
9 & 31 & 28.4 & 30.07 \\
10 & 24 & 24.2 & 26.11 \\
11 & 22 & 20.7 & 22.58 \\
12 & 22 & 17.7 & 19.49 \\
13 & 17 & 15.2 & 16.80 \\
14 & 16 & 13.1 & 14.47 \\
15 & 10 & 11.4 & 12.46 \\
\hline
\end{tabular}




\begin{tabular}{cccc}
\hline 16 & 8 & 9.8 & 10.73 \\
17 & 8 & 8.6 & 9.25 \\
18 & 11 & 7.5 & 7.98 \\
19 & 4 & 6.5 & 6.90 \\
20 & 3 & 5.7 & 5.97 \\
21 & 6 & 5.0 & 5.17 \\
$22-23$ & 9 & 8.3 & 8.39 \\
$24-25$ & 4 & 6.4 & 6.36 \\
$26-27$ & 7 & 5.1 & 4.85 \\
$28-30$ & 6 & 5.7 & 5.26 \\
$31-35$ & 8 & 6.1 & 5.26 \\
$36-48$ & 8 & 8.9 & 7.58 \\
\hline Total & 623 & 623.00 & 623.00 \\
\hline$\chi^{2}$ & & 19.81 & 15.33 \\
\hline$p$-value & & 0.8386 & 0.9645 \\
\hline
\end{tabular}

\section{CONCLUDING REMARKS}

Some important probabilistic properties, like infinite divisibility, have been derived for Kempton's full beta model. Although the probability mass function is complicated, its three-term recurrence relation facilitates computation and therefore its applications in statistical analysis. However, numerical stability of the recurrence formula is an issue. A heuristic method for testing forward stability of a three-term recurrence relation for probabilities have been presented. This heuristic rule allows the determination of the point where the recurrence relation should be started; it is easy to implement on the computer and does not require actual starting points of the recurrence formula. The implementation for the GLSD is similar. Four examples of data fitting for the FBM are also given to demonstrate its versatility and superiority to fit both short and moderately long-tailed data. The statistical literature is full of extensions and generalizations of basic count models like the Poisson and negative binomial distributions. It is shown that an existing model, the full beta model, can be used as a good empirical model.

\section{REFERENCES}

Bagnoli, M. \& Bergstrom, T. (2005). Log-concave probability and its applications. Economic Theory, 26, 445-469.

Bhati, D., Sastry, D.V.S., \& Qadri, P.Z.M. (2015). A new generalized Poisson-Lindley distribution: Applications and properties. Austrian Journal of Statistics, 44, 35-51.

Borzadaran, G. R. M. \& Borzadaran, H. A. M. (2011). Log-concavity property for some well-known distributions. Surveys in Mathematics and Its Applications, 6, 203-219.

Dallas, T.A., Deckber, R.R., \& Hastings, A. (2017). Species are not most abundant in the center of their geographic range or climatic niche. Ecology Letters, 20(12), 1526-1533.

Erdélyi, A. et al. (1953). Higher Transcendental Functions. Vol. I. McGraw Hill, New York. 
Feller, W. (1968). An Introduction to Probability Theory and its Applications. Vol I (3rd ed.). New York: John Wiley and Sons.

Fisher, R.A., Corbet, A.S., \& Williams, C.B. (1943). The relation between the number of species and the number of individuals in a random sample of an animal population. Journal of Animal Ecology, 12, 42- 58.

Garman, P. (1951). Original data on European red mites on apple leaves. Report. Connecticut.

Gomez-Deniz, E. \& Calderin-Ojeda, E. (2015). Parameter estimation for a new generalized geometric distribution. Communications in Statistics - Simulation and Computation, 44(8), 2023-2039.

Gupta R.C. \& Ong S.H. (2005). Analysis of long-tailed count data by Poisson mixtures. Communications in Statistics Theory and Methods, 34, 557573.

Holgate, P. (1970). The modality of some compound Poisson distributions. Biometrika, 57, 666-667.

Holla, M.S. \& Bhattacharya, S.K. (1965). On a discrete compound distribution. Annals of the Institute of Statistical Mathematics, 15, 377 - 384.

Karlis D. \& Xekalaki E. (2005). Mixed Poisson distributions. International Statistical Review, $73,35-58$

Kempton, R.A. (1975). A generalized form of Fisher's logarithmic series. Biometrika, 62, 29-38.
Maceda, E.C. (1948). On the compound and generalized Poisson distributions. Annals of Mathematical Statistics, 19, 414-416.

Ong, S.H. \& Lee, P.A. (1979). The noncentral negative binomial distribution. Biometrical Journal, 21, 611-628.

Ong, S.H., Lee, W. J., \& Low, Y.C. A general method of computing mixed Poisson probabilities by importance Manuscript submitted for publication.

Ong, S.H. (1995). Computation of probabilities of a generalized log-series and related distributions. Communications in Statistics - Theory and Methods, 24, 253 - 271.

Ong, S.H. \& Muthaloo, S. (1995). A class of discrete distributions suited to fitting very long-tailed data. Communications in Statistics - Simulation and Computation, 24, 929 - 946.

Press, W.H., Teukolsky, S.A., Vetterling, W.T., \& Flannery, B.P. (2007). Numerical Recipes. Cambridge, United Kingdom: Cambridge University Press.

Sichel, H.S. (1971). On a family of discrete distributions particularly suited to represent long-tailed frequency data. Proceedings of the Third Symposium on Maths. Statist., N.F. Laubscher (editor), 51 - 97. Pretoria, C.S.I.R. 
Steutel, F.W. (1983). Infinite divisibility. In S. Kotz, N.L. Johnson and C.B. Read (Eds.), Encyclopedia of Statistical Sciences 4. (pp. 114-116). New York: Wiley.

Steutel, F.W. \& Van Harn, K. (2003). Infinite Divisibility of Probability Distributions on the Real Line. New York: Marcel Dekker.

Taylor, P.J. (1967). Individual variations in sickness absence. British Journal of Industrial Medicine, 24, 169.
Tripathi, R.C., Gupta, R.C., \& White, T.J. (1987). Some generalizations of the geometric distribution. Sankhya B, 49, 218 -233 .

Walther, G. (2009). Inference and modeling with log-concave distributions. Statistical Science, 24(3), 319-327.

Weber, M.M., Stevens, R.D., DinizFilho, J.A.F., \& Grelle, C.E.V. (2017). Is there a correlation between abundance and environmental suitability derived from ecological niche modelling? a meta-analysis. Ecography, 40(7), 817-828. 